\title{
Reflexiones sobre el proceso de trabajo. Entrevista a Enrique de la Garza ${ }^{1}$
}

Pablo Seguel Gutiérrez

\section{APROXIMACIÓN AL TRABAJO DE ENRIQUE DE LA GARZA TOLEDO}

\subsection{El trabajo como operador analítico}

Desde mediados de la década de 1980 el trabajo de Enrique de la Garza Toledo se ha centrado en el estudio de las transformaciones recientes de las sociedades latinoamericanas en el contexto de la reconversión productiva, el cambio en las modalidades de inserción de los países en el mercado internacional y las transformaciones de la sociedad para afrontar este desafío. Para ello ha observado el mundo del trabajo desde diversos aspectos: desde el punto de vista de los mercados del trabajo, desde la perspectiva de la formación de subjetividades en los procesos de trabajo y de las relaciones entre economía, política y sociedad.

Enrique de la Garza Toledo es Doctor en Sociología por El Colegio de México y Posdoctorado de la Universidad de Warwick, Inglaterra, y de la Universidad de Berkeley, California, Estados Unidos. Ha publicado 18 libros sobre epistemología, sociología económica, historia del movimiento obrero y sociología del trabajo, además de coordinar innumerables textos académicos. Destacan entre sus trabajos la coordinación del Tratado latinoamericano de sociología del trabajo (2002), la coedición del Tratado de metodología de las ciencias sociales: perspectivas actuales, y su libro Reestructuración productiva, empresas y trabajadores en México (2006).

\subsection{Los perfiles y configuraciones sociotécnicas}

Una de las claves de análisis en su propuesta teórica la constituyen las nociones de perfil y de configuraciones sociotécnicas. Con esta noción el autor critica las teorías sobre la reestructuración productiva utilizadas para abordar los procesos de reconversión productiva en América Latina (teorías como el toyotismo, la clean production, la especialización flexible, el posfordismo, entre otras).

A su juicio, estas teorías carecen de evidencias empíricas verificables para América Latina, no establecen una relación clara entre las estructuras y los agentes y elaboran proyecciones en un alto nivel de abstracción que dificultan su utilización en los casos de estudio.

Esta entrevista se enmarca en las reflexiones e investigaciones efectuadas dentro del "Proyecto Bicentenario para el estudio de los movimientos sociales: memoria social y poder". Fue realizada en febrero de 2015 en México D.F. por Pablo Seguel Gutiérrez, estudiante de Licenciatura en Historia y de Sociología de la Universidad de Chile y asistente de investigación del Proyecto Bicentenario. 
Con la introducción de la noción de perfiles, De la Garza alude a la manera en cómo un caso empírico va constituyendo conceptos que, al articularse entre sí, conforman configuraciones, estableciendo relaciones teóricas con sustento empírico.

En específico, con la noción de configuración sociotécnica entiende la existencia de contradicciones entre los modelos productivos y las estrategias de desarrollo de diversos países, enfatizando los niveles de antagonismo existentes entre los agentes sociales sin que el conjunto de la configuración entre en crisis, adoptando perfiles acotados.

Con ello De la Garza destaca la idea de que el conflicto en los procesos de trabajo no necesariamente genera crisis, sino adaptación y oportunidades de transformaciones. Por otra parte, marca la importancia de las relaciones entre los agentes de una configuración, los cuales establecen vínculos de subordinación, dependencia, acuerdos y desacuerdos.

\subsection{Revitalización del debate acerca del proceso de trabajo}

Su enfoque de análisis se ha centrado en la observación de los procesos de trabajo que van constituyendo perfiles determinados y configuraciones sociotécnicas. Para ello retoma la noción desarrollada por Karl Marx e introducida en el debate académico en la década de 1970, ampliándola hacia el estudio de las tendencias contemporáneas de la economía internacional. Su foco ha sido estudiar los procesos de trasformación económica desde la perspectiva de los agentes y las relaciones que estos establecen en los procesos de trabajo en específico con los entornos socioambientales y con el conjunto de instituciones jurídicas y políticas que las regulan.

Con estos elementos ha podido poner el acento en los procesos de subjetivación de los agentes involucrados en los procesos de trabajo, los cuales, evaluando el entorno socioambiental y jurídico-político, desarrollan formas de racionalización y adaptación que les permiten construir estrategias de subsistencia económica, conflictividad social y sobrevivencias que forman perfiles de desarrollo específicos.

Además de aquello, plantea varios retos para abordar lo que Marx denominó producción inmaterial, que supone un desafío de consideración para el estudio de los procesos de trabajo en el sector de servicios.

\section{ENTREVISTA}

En su artículo del 2012 sobre la revitalización del debate del proceso de trabajo, usted da algunas pistas desde donde teóricamente la sociología debiera aproximarse al estudio de este tema, ¿cuáles son a su juicio los elementos indispensables para el estudio del proceso de trabajo?

Mi punto de partida ha sido siempre la sección cuarta de El Capital de Marx, cuando dice: "el proceso de producción capitalista se desglosa en un proceso de valorización, de generación de valor, y un proceso de trabajo. Motivo por el cual el capital, para cumplir su 
función de explotación, de apropiación de los valores generados por el trabajo, tiene que controlar a la fuerza de trabajo en el proceso, es decir, a los trabajadores. De tal manera que el proceso de trabajo y el proceso de valorización van de la mano, no siendo reducibles uno al otro". Esto quiere decir que se pueden conseguir ciertos niveles de extracción de plusvalías de diferentes formas por parte del capital, de tal manera que el solo hecho de hablar de explotación, el nivel de valorización, no nos dice nada. No se puede deducir nada a partir de esto. No se puede deducir cuáles son las formas concretas de cómo se realiza el trabajo. Para ello tengo que investigar en completo el proceso de trabajo, tomando en consideración que no podemos reducirlo solo a factores de la producción, sino que verlo como una interrelación social de los hombres, con ellos mismos y con los medios de producción, es decir, interacciones que conllevan significados y que están inmersos en relaciones de poder.

En la sociología del trabajo clásica, el concepto de control ha sido el concepto clave para referirse a esta problemática. En específico en su versión americana con Harry Braverman y en la versión francesa de George Friedman y Pierre Naville. Ambas reflexiones partieron en una época en donde la influencia del marxismo era significativa en la sociedad, pero no en los circuitos académicos. Con posterioridad vinieron otras perspectivas que no han coincidido con esto, incluso que han negado la importancia del control en el proceso de trabajo. Actualmente hay perspectivas de este tipo, donde el control pierde pertinencia; ya no es importante controlar porque, como señalan algunos autores, los nuevos trabajadores han ido hacia una sumisión voluntaria, que no necesitan ser controlados, ya que voluntariamente aceptan su condición, lo que es muy discutible.

Hay corrientes que han negado lo que los clásicos han planteado, que es el tema del control. Entonces yo siempre empiezo por ahí, en el tema del control, donde se observan interacciones, pero también significados, sentidos, formas de representación y elaboración simbólica mediadas por sujetos; de tal manera que la parte material, incluyendo lo económico, no puede desligarse en el proceso de trabajo de la parte subjetiva, que serían todas las concepciones que tienen los trabajadores y mandos medios, hasta la propia gerencia, sobre el trabajo, sobre la empresa, sobre lo que es negociable, lo que no es negociable. Es decir, el proceso de trabajo en sus resultados no es determinístico, como en la economía donde la suma de costos define cuánto se va a producir en valor.

En la perspectiva que parte del marxismo, el proceso de trabajo puede tener diferentes resultados en valorización dependiendo de relaciones de fuerza, acuerdos, conflictos que se den en el propio trabajo, los que forman diferentes perfiles. Esto supone la intervención de actores del proceso de trabajo que no están determinados solo porque se les paga cierto salario, sino porque tienen ciertas concepciones, las cuales están vinculadas a las formas de organizar el trabajo, con cierta tecnología, en ciertas relaciones laborales formales e informales.

Además esta fuerza de trabajo tiene ciertas características sociodemográficas, puede haber más mujeres, hombres, jóvenes, viejos, ciertas características en sus calificaciones, etc. Por este motivo, la conexión con el concepto de configuración sociotécnica no es algo al margen o por detrás de los procesos de trabajo. Por el contrario, significa pensar que el 
proceso de trabajo se realiza dentro de ciertas configuraciones, cierto nivel tecnológico, cierto tipo de organización, ciertas relaciones laborales, cierto perfil de mano de obra y ciertas culturas del trabajo.

Digamos que la configuración es un nivel estructural pero no sistémico, es una plataforma dentro de la cual los actores del trabajo actúan, interaccionan y producen parte de sus actuaciones, de tal manera que no todo lo que pasa en la empresa lo define la configuración. Más bien es el marco en el cual se realizan las acciones, es decir, el paso siguiente después de definir en qué marco o configuración estamos interaccionando, es decir, el escenario de los propios trabajadores y mandos medios en sus interacciones, en sus conflictos o no conflictos, acuerdos, desacuerdos, en qué influyen en los resultados de la producción, pero también en términos de identidades, de constitución de sujetos. El concepto de configuración sociotécnica es una ampliación para el análisis del proceso de trabajo.

Usted señala que el libro de Harry Braverman, "Trabajo y capital monopólico", publicado en 1974, inaugura el debate sobre el proceso de trabajo en la tradición marxista. En el mismo libro, Braverman señala que el marxismo ha descuidado el estudio en específico del proceso de trabajo. ¿A qué se debe este descuido?

Digamos que esa ha sido una observación propia de esa época. Estamos hablando de un libro que se publicó en el 70 y cuya validez se acota a la realidad de los procesos de trabajo de esa época. No se puede afirmar que el marxismo se olvidó simplemente, porque de ahí surgió el debate del proceso de trabajo en donde los teóricos marxistas se introdujeron plenamente al análisis del proceso de trabajo. Esto a pesar de que en la obra de Marx, en la sección cuarta de El Capital, el planteamiento ya estaba desarrollado. En esta sección Marx señala que el proceso de trabajo se da simultáneamente al proceso de valorización, por lo que en los procesos de trabajo se generan valores y relaciones sociales, las que implican poder, significados, que se intercambian, relaciones de fuerza, resistencia y control.

Entonces todo eso ya estaba en Marx; lo que sucedió fue que el marxismo durante mucho tiempo no lo desarrolló. Curiosamente los primeros que retomaron esto, antes que Braverman, fueron George Friedman y Pierre Naville. Ambos, al igual que Braverman, estaban al tanto de este debate al interior del marxismo, pero no seguían la trayectoria que estaba siguiendo en general el marxismo en la posguerra. Si esto es así, ¿por qué durante tantos decenios los marxistas se olvidaron del proceso de trabajo? Yo diría que por un materialismo y un economicismo mal entendido, es decir, bastaba con estudiar el nivel que Marx Ilama de la valorización para que todo se desprendiera de ahí. Del proceso de trabajo se desprendía el Estado, el derecho, la cultura, todo; es decir, no había que estudiarlo en particular, sino que era una consecuencia. Entonces predominó un marxismo muy economicista, donde el nivel de la valorización era suficiente, muy autosuficiente para explicar toda la sociedad.

Esta interpretación tiene como correlato el sostenimiento de que no existen autonomías relativas entre las diversas esferas sociales y metodológicamente implica que no es necesario estudiarlas en específico. Esta mala interpretación originó un marxismo de la Economía 
Política, pero no del proceso de trabajo. Así sucedió con otros temas, como por ejemplo con la noción de cultura. Entonces, los innovadores como Gramsci lo que hicieron fue meterse en la superestructura y, sin desconocer la existencia de la economía, trataron de explicarla en su dinámica interna. Esto les permitió crear nuevos conceptos y cambiar las implicancias teóricas de otros, como por ejemplo el concepto de hegemonía. La noción de hegemonía trabajada por Gramsci no es la misma que la trabajada por Lenin. El concepto de sociedad civil de Gramsci no es el mismo que consideraba Marx, pero eso fue gracias a la elaboración teórica y al no contentarse con decir que era consecuencia de la economía.

Algo similar sucedía con el proceso de trabajo. No era importante estudiarlo porque lo políticamente relevante era que la clase obrera ya era explotada, producía plusvalía y lo único que le faltaba era conciencia de clase, la cual le tenía que venir desde afuera, que era un planteamiento propio del marxismo-leninismo. Pero la clase obrera estaba generando formas de conciencia y subjetividad en el trabajo y fuera del trabajo que ponían en cuestión este supuesto. Además de que esto tiene que ver con la manera de trabajar, en el cómo se vinculaba toda la configuración sociotécnica. En todo esto el marxismo se quedó muy rezagado, retomándolo con mucho retraso en el caso de Braverman. En parte por el contexto político, como por el idioma en el que escribió, porque publicó su trabajo en inglés y esto redundó en que su trabajo repercutió con fuerza en el mundo académico-intelectual anglosajón.

Braverman le dio más proyección a este debate que los franceses como Friedman, que escribieron antes. Lamentablemente el francés nunca ha sido una lengua con tanto impacto en el debate internacional como el inglés, por eso fue tan fuerte el impacto de Braverman a pesar de que había antecedentes en Francia y en Italia. En Italia Raniero Panzieri, diez años antes que Braverman, ya había planteado algo similar. Al igual que el francés, el italiano es un lenguaje con poco impacto internacional, salvo en la Ciencia Política. Los autores italianos son poco traducidos, no son leídos en su lenguaje de origen. Por estos motivos Panzieri tuvo poco impacto a pesar de haberlo planteado con mucha claridad. La irrupción de la propuesta de Braverman marcó todo un revuelo académico-intelectual en un contexto postmayo de 1968, de tal manera que profesores y estudiantes estaban receptivos a los debates de la teoría crítica, en especial del marxismo. Una situación que en la década de 1950 era impensable al menos en Estados Unidos, es decir, si Braverman hubiera escrito en la década de 1950 su impacto hubiera sido menor del que tuvo.

¿Desde qué lugar surge la importancia de atender a la subjetividad en el proceso de trabajo?, ¿es un remanente dado a partir del debate abierto entre los Estudios Críticos de Gestión Empresarial y la sociología inglesa del proceso de trabajo o tiene un lugar propio en términos teóricos?

Tiene un lugar propio en términos teóricos. Es decir, se puede entrar al debate con el Critical Management, pero el interés no surgió de un debate con ellos. Tiene sus propios antecedentes en los trabajos de Marx, en donde señala en una de las tesis sobre Feuerbach que "el viejo materialismo se ha quedado corto, porque solo se ha fijado en la cara objetiva del objeto, léase en la parte economía, pero ha dejado muy descuidado el materialismo a la 
cara subjetiva del objeto, dejándoselo al idealismo"2 ${ }^{\prime 2}$ Con esta tesis Marx estaba haciendo una observación de total actualidad. Fueron los marxistas quienes durante muchos decenios se conformaron con el estudio de la cara objetiva del objeto, con el análisis de la Economía Política. Por el contrario, la cara subjetiva del proceso referido a la subjetividad y a los procesos de subjetivación quedaron reducidos a la idea de la verdadera/falsa conciencia, de tal manera que los obreros, sin la intervención/mediación de partidos o intelectuales colectivos, no se subjetivaban en un sentido verdadero, no eran capaces de tomar conciencia sobre su propia situación. En ese sentido, ipara qué investigar, si solo basta con que llegue un partido, imbuido de una conciencia teórica correcta sobre la situación de los trabajadores?

Queda en evidencia que esta era una operación mecánica, donde no valía la pena detenerse en la parte subjetiva. Esta proposición propia del análisis marxista-leninista impidió que durante años la teoría crítica marxista se abocara a este debate. Desde finales del siglo XIX y comienzos del XX quienes desarrollaron reflexiones sobre la subjetividad fueron las corrientes hermenéuticas, el historicismo de Wilhelm Dilthey, la fenomenología de Alfred Schütz, las corrientes interpretativas del discurso, el existencialismo de Jean Paul Sartre. Había una enorme cantidad de producción teórica en relación a este tema, mientras que el marxismo hegemónico de la Unión Soviética quedó a la saga.

Esto no quiere decir que todo el marxismo apuntó en esa dirección. El marxismo occidental en buena parte se interesó en este debate, pero fue marginalizado por efecto de un factor político importante. La hegemonía de la Unión Soviética dificultó que el desarrollo de teorías críticas sobre la cultura y la subjetividad tuvieran un impacto mayor, como le sucedió a la Escuela de Frankfurt y a la Escuela de Budapest. Pero no solo ellos, Gramsci fue marginalizado, Walter Benjamin terminó suicidándose, Lukács produjo su obra en la marginalidad, Ágnes Heller, Thompson y muchos más. Todos aquellos que se interesaban por la dimensión cultural de los fenómenos humanos, por la subjetividad, por los problemas más finos del poder, de la dominación, fueron marginalizados. El grueso del marxismo seguía siendo un marxismo soviético, mecanicista y economicista, sin mucho desarrollo en estas temáticas. Cuando se abren las ventanas en la década de 1970, al calor de los movimientos estudiantiles y otros acontecimientos políticos, los marxistas no ortodoxos comienzan a interesarse por los temas de la subjetividad. En ese contexto, una de las líneas de desarrollo fue el de la subjetividad y el trabajo. Esto quiere decir que los obreros, sean como sean, siempre tienen alguna forma de subjetividad, construyen significados en el proceso de trabajo, de tal manera que la investigación del proceso de trabajo no puede ser solo la parte material si no también la parte subjetiva.

2 Se refiere a la Tesis I sobre Feuerbach: "El defecto fundamental de todo el materialismo anterior -incluido el de Feuerbach- es que solo concibe las cosas, la realidad, la sensoriedad, bajo la forma de objeto o de contemplación, pero no como actividad sensorial humana, no como práctica, no de un modo subjetivo. De aquí que el lado activo fuese desarrollado por el idealismo, por oposición al materialismo, pero solo de un modo abstracto, ya que el idealismo, naturalmente, no conoce la actividad real, sensorial..." (consultada en: www.marxists.org/espanol/m-e/1840s/45-feuer.htm). 
Con posterioridad se abre el debate con el Critical Management, pero el debate inicial se da con las corrientes toyotistas. El management en la ideología liberal se presenta como con cara amable, se trata de tener obreros participativos, recoger sus opiniones, sus experiencias, permitirles opinar, hacer no tan vertical las decisiones. Se busca ante todo crear una identificación con la empresa, de tal modo de propender hacia una subjetivación que contemple que el desarrollo y buen manejo de la empresa depende de las labores de cada uno de los obreros y trabajadores de esta. Ahí la discusión sobre la subjetividad en el debate del proceso de trabajo se da también con el management, que habla a su manera de una subjetividad a favor de la empresa. Pero en estricto rigor viene de la apertura del marxismo, así como la entrada del debate sobre el proceso de trabajo

Con este debate se buscó remarcar la idea de que el proceso de trabajo no era solo las partes materiales, sino que también las subjetivas y entonces la apertura tenía que continuar hacia eso. En el camino se encuentran con el toyotismo, se debate con él. En el camino se siguió debatiendo con muchos otros y hasta la actualidad en los debates del proceso de trabajo el tema de la subjetividad e identidad es lo más importante porque se implica con otra polémica que no es el management, sino que son las teorías del fin del trabajo, del declive de la sociedad salarial, de la pérdida de peso estratégico de la clase obrera en la sociedad.

Estos debates surgieron en la década de 1980, de modo no muy desarrollado en el tema de la subjetividad. Su centro más bien eran las ocupaciones, afirmando que estas se habían vuelto tan heterogéneas que ya no había posibilidades de identificación de unas con otras. El debate que en la década siguiente tuvo mayor impacto lo introdujo Zygmunt Bauman, quien se constituyó en el teórico de la fermentación de las identidades.

Bauman proponía que las biografías, las que son muy líquidas, impiden la formación de identidades colectivas estables, entonces ahí es donde realmente las teorías del proceso de trabajo entran a discutir sobre el tema de la identidad con propiedad. Es decir, con teorías que ya trataban sobre la identidad, aunque no lo refirieran al trabajo necesariamente; con teorías sociales que no se interesaban en el trabajo necesariamente sino en la identidad. Entonces, ¿cómo se constituyen las identidades? Se constituyen nada más porque se tienen las mismas ocupaciones laborales, lo que también es muy mecanicista.

Afirmar que la ocupación laboral determina la identidad y, como consecuencia de esto, su posicionamiento político, no explica nada. No es capaz de explicar cómo movimientos sociopolíticos que remecieron sociedades enteras en América Latina se conformaron a partir de ocupaciones tan heterogéneas, como obreros, campesinos, artesanos. ¿Cómo este arco de ocupaciones tan heterogéneo crea identidades? Esto no lo podía responder Bauman. Entonces eso le dio mucha riqueza a las teorías del proceso de trabajo porque implicó ponerse en comunicación con otras teorías que no eran del proceso de trabajo, que hablaban de identidad.

Como resultado el enriquecimiento fue grande en el debate con las fragmentaciones de Bauman, con el aporte de la cultura obrera, sobre todo con los historiadores. E.P. Thompson ya la había introducido, pero no había desarrollado esta discusión tan a fondo, como sucedió 
en los 90 y al inicio del siglo XX. De estos debates resultaron teorías más sofisticadas, en comunicación con otros ámbitos de teorización que no son del trabajo y que son necesarios, ya que no todo puede salir de las teorías del proceso de trabajo.

En la actualidad, ¿cómo se podría abordar el problema de la politización en un contexto de transformaciones de las formas clásicas del trabajo y también de las formas de organización de los trabajadores? A su juicio, ¿cómo se expresa el campo de la politización en el proceso de trabajo, en las condiciones actuales del trabajo?

Una complicación que vino en el camino en los años 60, 70, y que no se avizoraba con claridad, fue la tercerización, no de subcontratación, sino la de la ampliación del sector terciario del servicio, situación que se dio muy fuerte a nivel mundial, de tal manera que los servicios pasaron a llevar la pauta del desarrollo capitalista en términos económicos y la industria quedó relativamente rezagada, cosa que nadie se imaginaba sobre todo en los 70. Esta nueva circunstancia de la importancia de los servicios en el desarrollo económico obligó a toda una reformulación de los estudios del proceso de trabajo.

En muchos de los servicios está directamente implicado el cliente mientras se presta el servicio, cosas que en las fábricas no suceden. Muchos servicios son de intercambio simbólico, no hay producto material de por medio, entonces eso lleva a muchas propuestas que son el gran tema de la actualidad: ¿cómo abordar este sector de los servicios que ahora se vuelve el más importante en la ocupación?, ¿qué tanto son suficientes las categorías del proceso de trabajo desarrolladas en la época industrial? Por ejemplo el taylorismo es una categoría que surgió para cierto tipo de industrias, que no se dan con fuerza hoy y que no se adecuan a la realidad. ¿Qué se hace por ejemplo en este restaurante?, ¿hay taylorismo?, ¿no hay taylorismo?, ¿hay que pensar en otras categorías?

Esa es la gran temática de las discusiones sobre el proceso de trabajo, es decir, ¿cómo abordar los procesos de trabajo de servicio, cuando las categorías fueron acuñadas para la industria? Entonces hay muchas propuestas y este es el debate más fructífero. Ya no es tanto el debate para la industria, no quiere decir que la industria no esté analizándose, pero con todos los cambios tecnológicos en la industria, la gran novedad es ¿cómo abordar los servicios?

Hay una complejidad mayor, porque el producto no es algo que se pueda tocar, es algo puramente simbólico, entonces se puede tratar en el proceso de trabajo de diseñar un producto, un servicio, o bien una mercancía como un automóvil. Pero no es así en el servicio de un restaurante, donde hay una parte material que es donde elaboran los alimentos, pero hay otra parte que es el trato y el servicio del mesero que es interactivo. Entonces en el restaurante se paga la interacción, el prestigio y la distinción, no solamente la hamburguesa. Se consumen ciertos tipos de productos, no solo por el placer y la necesidad de alimentación, sino que por el estatus y la distinción adjunta al consumo de ciertos productos y servicios.

En esto la producción simbólica y de valores se torna fundamental cuando el cliente es un componente de primera línea en el proceso de trabajo. En estos trabajos el control lo ejerce la gerencia o bien hay respuestas de los trabajadores, pero también hay control 
del usuario o cliente. Hay servicios donde los clientes tienen que hacer ciertas actividades, por ejemplo en un supermercado grande el cliente tiene que arrastrar sus carros, poner sus mercancías, Ilevarlo hasta la caja registradora, entonces se puede hablar de trabajo del cliente. Se puede hablar de anomalías respecto del trabajo clásico, esa es la discusión de la actualidad. Es decir, con qué categorías analizar el trabajo de los servicios, el trabajo que se da en los espacios públicos.

Por ejemplo, el chofer de un bus del transporte público no solo tiene que lidiar con el cliente que se sube, sino con peatones, automóviles, con agentes del tránsito que no son agentes vigilantes de las condiciones laborales, sino del tránsito. Entonces estos, ¿influyen su trabajo? Esto implicaría que en el espacio público, ¿el concepto de control tiene que ser multidimensional?, es decir ¿muchos actores?, ¿no solo los clientes?, ¿qué pasa con el concepto de relación laboral?

El concepto clásico que se consolidó fue el de obrero, asalariado y empleado, pero si hilamos fino el concepto más básico es por la relación social que se da en el trabajo. Entonces en el trabajo la relación que tenemos aquí en este restaurante también es con los meseros, aunque sea superficial. Entonces cuando les Ilamamos la atención, le llamamos que venga, estamos hasta cierto punto controlándolo y la relación puede ser cordial, de sonrisas o puede ser agria, incluso uno puede quejarse con el gerente de aquí y puedes decir estoy siendo maltratado, entonces, ¿hasta qué punto la relación laboral tiene que ser ampliada?

Eso se puede extender a los trabajadores informales de la calle, el que vende en la calle, el que hace comida en la calle, entonces ese es el gran tema del momento de los estudios del trabajo. Es todo un reto porque implica una revisión a fondo de las teorías sobre proceso de trabajo, no quiere decir que la relación capital-trabajo no existe. Sigue existiendo, en términos de valorización, pero en términos de proceso de trabajo, ¿qué implica ser mesero con relación a obrero de un ensamble automotriz?, ¿es lo mismo exactamente como proceso de trabajo? No, empezando porque el cliente no está dentro de las líneas automotrices, de producción, nunca los dejan entrar. En cambio en estos servicios (restaurante) si uno no está aquí no hay servicio, no hay venta en el restaurante si no estamos nosotros, somos una parte integrante del proceso de trabajo.

Marx ya lo había notado. Hay tres referencias claves, uno son los Grundrisse, otro es en el capítulo sexto inédito de El Capital y el otro es la historia crítica de las teorías de la plusvalía. Entonces en esos tres textos Marx percibe que hay otros tipos de producciones, que corresponderían a los servicios: en una obra de teatro capitalista (el dueño es el capitalista) el empleado es el actor o el principal, y ahí se genera una obra de teatro que tiene la particularidad respecto de la producción material, que al mismo tiempo que se está generando la obra de trabajo, está circulando por el público y se está consumiendo por el público. Es decir, lo que en la producción material aparecen como etapas separadas en el tiempo, en estos servicios aparecen comprimidas al mismo tiempo, es decir, el espectador se consume en su subjetividad en la obra de teatro, circulan del escenario hasta él y a eso le da un nombre, le llama la producción inmaterial. Es decir, Marx se dio perfectamente cuenta de estos procesos de trabajo, lo que pasa es que no logró prever el capitalismo industrial 
que iba en ascenso. Había empezado la revolución industrial y ese ascenso se mantuvo durante los años 50, pero con posterioridad tendrá un viraje hacia los servicios. Entonces ahí es donde viene el problema.

Desde este punto de vista, los problemas de la politización en los procesos de trabajo es una interrogante abierta. Un desafío que tenemos que explorar a base de investigación empírica y aportes teóricos, en consideración a las transformaciones contemporáneas de los procesos de trabajo.

Si usted tuviese que hacer una enumeración de temas de investigación y orientaciones actuales de los estudios del trabajo en América Latina, ¿cuáles señalaría que son estos?

El gran tema de investigación es, ¿cómo abordar el estudio del proceso de trabajo en servicios en donde está el cliente, en donde lo que se producen son símbolos o interacciones?, ¿con que categorías?, ¿qué relación tiene esto con el tema de la valorización?, ¿hasta qué nivel ampliar el concepto de mercancía y producción inmaterial?, y ¿ hasta qué punto puede una gerencia controlar estrictamente el trabajo en estos servicios? Es decir, ¿qué tanto el trabajador que interacciona con el cliente está controlado o tiene mucho más margen de acción que el de una fábrica?

Por ejemplo en los call center hay instrucciones claras hacia los jóvenes que trabajan ahí para que la conversación sea muy escueta. Sin embargo, hay veces que el cliente se sale del guion y comienza a plantear otros temas, entonces ¿qué tiene que hacer? Tampoco los puede cortar, entonces, ¿qué tan controlados pueden ser este tipo de trabajo con consideración de los materiales?

El máximo nivel de este tipo de control se consideró que estaba expresado con el taylorismo. Pero ¿qué tanto pueden ser taylorizados los trabajadores? Sí hay intentos sistemáticos que apuntan hacia ese objetivo, pero en el camino se encontraron muchos obstáculos, porque no es lo mismo controlar a tu dependiente que controlar al cliente, porque el cliente no es un factor constante, entonces son grandes temas.

Otro tema lo es por ejemplo el sector servicios. El trabajo de los servicios, ¿qué tanto se presta para la creación de identidad colectiva?, ¿o cuáles son los determinantes de las posibles identidades colectivas? Entonces en el mundo desarrollado, especialmente en Europa, yo diría que los temas más importantes tienen que ver con la dimensión de los servicios, en una ampliación del concepto de trabajo. Por ejemplo, zes trabajo el trabajo de los líderes de un partido político? Hay análisis empíricos de cómo realizan su trabajo, para los cuales tampoco nos es útil la noción de trabajo que se desprende del taylorismo. Entonces, ¿con qué concepto los estudiamos?

Hay en ese sentido una clara tendencia en los estudios del trabajo que busca una ampliación del ámbito de lo que es la actividad laboral y la ampliación más importante es por el lado de los servicios. Sin embargo, esto también se lleva a las fábricas, porque en las fábricas también hay servicios llamados productivos, los servicios de contabilidad, diseño, mercadeo, que se les da poca importancia. También la subcontratación y las nuevas formas de vínculo 
laboral, el trabajo por honorarios, a tiempo parcial, por estación, por subcontratación, que algunos Ilaman las zonas oscuras de la asalarización de la población, entonces yo creo que este es otro de los grandes temas, que incluye por supuesto la industria.

\section{BIBLIOGRAFÍA}

De la Garza, E. (coord.) (2002): Tratado latinoamericano de sociología del trabajo, Fondo de Cultura Económica, México D.F.

(2006): Restructuración productiva, empresas y trabajadores en México, Fondo de Cultura Económica, México D.F.

(2012): "La revitalización del debate del proceso de trabajo", Revista Latinoamericana de Estudios del Trabajo, 16 (26), pp. 7-35.

De la Garza, E. y G. Leyva (eds.) (2012): Tratado de metodología de las ciencias sociales: perspectivas actuales, Fondo de Cultura Económica, UAM-I, México D.F.

Recibida: 22-06-2015

Aceptada: 30-06-2015 